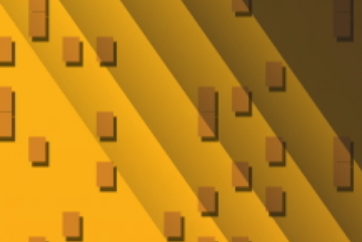

$$
\begin{aligned}
& \text { AVgebra \& } \\
& \text { Number } \\
& \text { Theory } \\
& \text { Volume } 5
\end{aligned}
$$

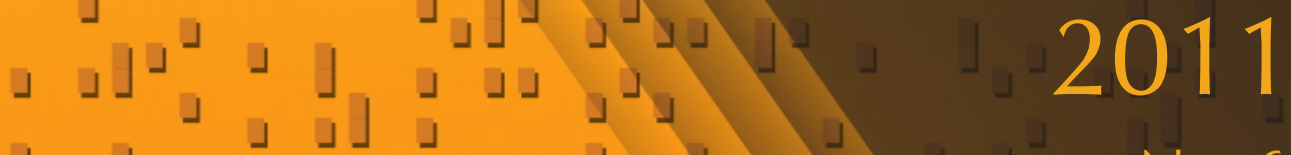

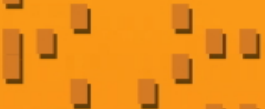

$$
\begin{aligned}
& \lrcorner\lrcorner
\end{aligned}
$$

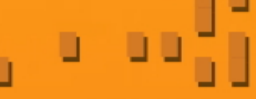

$$
\begin{aligned}
& \begin{array}{l}
\lrcorner \\
\lrcorner
\end{array} \\
& \lrcorner\lrcorner\lrcorner\lrcorner \\
& \lrcorner \\
& \text { No. } 6
\end{aligned}
$$

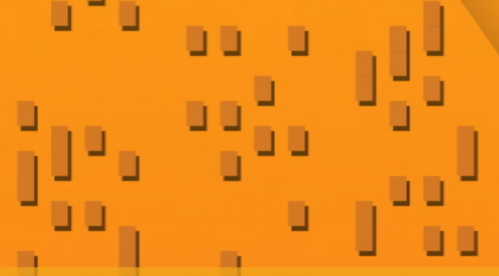

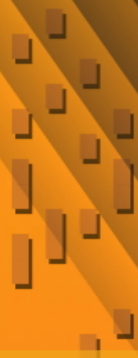

Sur le groupe de Chow de codimension deux des variétés sur les corps finis

Alena Pirutka

」」 


\title{
Sur le groupe de Chow de codimension deux des variétés sur les corps finis
}

\author{
Alena Pirutka
}

En utilisant la construction de Colliot-Thélène et Ojanguren, on donne un exemple d'une variété projective et lisse géométriquement rationnelle $X$, définie sur un corps fini $\mathbb{F}_{p}$, telle que d'une part le groupe $H_{\mathrm{nr}}^{3}(X, \mathbb{Z} / 2)$ est non nul et, d'autre part, l'application $C H^{2}(X) \rightarrow C H^{2}\left(X \times_{\mathbb{F}_{p}} \overline{\mathbb{F}}_{p}\right)^{\operatorname{Gal}\left(\overline{\mathbb{F}}_{p} / \mathbb{F}_{p}\right)}$ n'est pas surjective.

Using a construction of Colliot-Thélène and Ojanguren, we exhibit an example of a smooth projective geometrically rational variety $X$ defined over a finite field $\mathbb{F}_{p}$, such that the group $H_{\mathrm{nr}}^{3}(X, \mathbb{Z} / 2)$ is nonzero and the map $C H^{2}(X) \rightarrow$ $C H^{2}\left(X \times_{\mathbb{F}_{p}} \overline{\mathbb{F}}_{p}\right)^{\mathrm{Gal}\left(\overline{\mathbb{F}}_{p} / \mathbb{F}_{p}\right)}$ is not surjective.

Soit $\mathbb{F}_{p}$ un corps fini de cardinal $p$. Soit $\overline{\mathbb{F}}_{p}$ une clôture algébrique de $\mathbb{F}_{p}$ et soit $G=\operatorname{Gal}\left(\overline{\mathbb{F}}_{p} / \mathbb{F}_{p}\right)$ le groupe de Galois absolu. Soit $X$ une $\mathbb{F}_{p}$-variété projective et lisse, géométriquement connexe, de dimension $d$ et soit $\bar{X}=X \times_{\mathbb{F}_{p}} \overline{\mathbb{F}}_{p}$. On dispose d'une application naturelle

$$
C H^{i}(X) \rightarrow C H^{i}(\bar{X})^{G}
$$

entre les groupes de Chow des cycles de codimension $i$ sur $X$ (resp. sur $\bar{X}$ ) modulo l'équivalence rationnelle. Cette application est surjective pour $i=0,1, d$ (voir remarque 3.1). On peut se demander s'il en est ainsi pour $2 \leq i<d$.

Dans cet article, on donne un contre-exemple pour $i=2$. Dans ce cas, des arguments de $K$-théorie algébrique [Kahn 1996] permettent de faire un lien entre le conoyau de l'application $\mathrm{CH}^{2}(X) \rightarrow C H^{2}(\bar{X})^{G}$ et le groupe de cohomologie non ramifiée $H_{\mathrm{nr}}^{3}\left(X, \mathbb{Q}_{l} / \mathbb{Z}_{l}(2)\right)$. On montre dans la $2^{\text {ème }}$ partie qu'il suffit d'assurer que ce dernier groupe est non nul. Pour ce faire, les techniques développées par Colliot-Thélène et Ojanguren [1989] sont disponibles. En utilisant leur méthode, on construit ainsi dans la $3^{3 e ̀ m e}$ partie une variété projective lisse $X$ géométriquement connexe définie sur un corps fini $\mathbb{F}_{p}$ convenable, telle que

$$
\text { l'application } \mathrm{CH}^{2}(X) \rightarrow C H^{2}(\bar{X})^{G} n^{\prime} \text { 'est pas surjective. }
$$

MSC2000: 14C25.

Mots-clefs: groupes de Chow, cohomologie non ramifiée, Chow groups, unramified cohomology. 
Plus précisément, $X$ est une variété géométriquement rationnelle de dimension 5 , admettant un morphisme vers $\mathbb{P}_{\mathbb{F}_{p}}^{2}$ à fibre générique une quadrique lisse «voisine» de Pfister. Notre méthode permet d'obtenir de tels exemples sur des corps finis $\mathbb{F}_{p}$ pour une infinité de nombres premiers $p$.

\section{Notations et rappels}

Notations. Étant donné un corps $k$, on note $k^{*}$ le groupe multiplicatif $k-\{0\}, \bar{k}$ une clôture séparable de $k$ et $G=\operatorname{Gal}(\bar{k} / k)$ le groupe de Galois absolu. On note $\mathbb{F}_{p}$ le corps fini de cardinal $p$.

Si $X$ est une variété algébrique définie sur un corps $k$, on note $\bar{X}=X_{\bar{k}}=X \times{ }_{k} \bar{k}$. Si $X$ est intègre, on note $k(X)$ son corps des fractions et si $X$ est géométriquement intègre, on note $\bar{k}(X)$ le corps des fractions de $\bar{X}$. On dit que $X$ est $k$-rationnelle si $X$ est birationnelle à $\mathbb{P}_{k}^{n}$ et on dit que $X$ est géométriquement rationnelle si $\bar{X}$ est $\bar{k}$-rationnelle.

Pour une $k$-variété intègre $X$ et $i$ un entier, on note $X^{(i)}$ l'ensemble des points de $X$ de codimension $i$ et on note $C H^{i}(X)$ le groupe des cycles de codimension $i$ modulo l'équivalence rationnelle.

Si $A$ est un groupe abélien et $n$ est un entier, on note $A[n]$ le sous-groupe de $A$ formé par les éléments annulés par $n$. Pour $l$ un nombre premier, on note $A\{l\}$ le sous-groupe de torsion $l$-primaire.

Pour $M$ un $G$-module continu discret on note $H^{i}(k, M)=H^{i}(G, M)$ le $i$-ème groupe de cohomologie galoisienne et on note $M^{G}=H^{0}(k, M)$ le sous-groupe formé par les éléments invariants par $G$.

Rappels de cohomologie étale. Étant donnés un corps $k$ et un entier $n$ inversible sur $k$, on note $\mu_{n}$ le $k$-schéma en groupes (étale) des racines $n$-ièmes de l'unité. Pour $j$ un entier positif, on note $\mu_{n}^{\otimes j}=\mu_{n} \otimes \cdots \otimes \mu_{n}$ ( $j$ fois). On pose $\mu_{n}^{\otimes j}=$ $\operatorname{Hom}_{k-g r}\left(\mu_{n}^{\otimes(-j)}, \mathbb{Z} / n\right)$ si $j$ est négatif et $\mu_{n}^{\otimes 0}=\mathbb{Z} / n$. Ces $k$-schémas en groupes donnent des faisceaux étales, notés encore $\mu_{n}^{\otimes j}$, sur toute $k$-variété $X$. On note $H^{i}\left(X, \mu_{n}^{\otimes j}\right)$ les groupes de cohomologie étale de $X$ à valeurs dans $\mu_{n}^{\otimes j}$. Lorsque $n=2$, on a un isomorphisme canonique $\mu_{2}^{\otimes j} \stackrel{\sim}{\rightarrow} \mathbb{Z} / 2$ pour tout $j$.

Définition 1.1. Pour $X$ une $k$-variété intègre, un entier naturel $j \geq 1$ et $i \in \mathbb{Z}$ un entier relatif, on définit les groupes de cohomologie non ramifiée

$H_{\mathrm{nr}}^{j}\left(X, \mu_{n}^{\otimes i}\right) \stackrel{\text { déf }}{=} H_{\mathrm{nr}}^{j}\left(k(X) / k, \mu_{n}^{\otimes i}\right)=\bigcap_{A} \operatorname{Ker}\left[H^{j}\left(k(X), \mu_{n}^{\otimes i}\right) \stackrel{\partial_{j, A}}{\rightarrow} H^{j-1}\left(k_{A}, \mu_{n}^{\otimes i-1}\right)\right]$.

Dans cette formule, $A$ parcourt les anneaux de valuation discrète de rang un, de corps des fractions $k(X)$, contenant le corps $k$. Le corps résiduel d'un tel anneau $A$ est noté $k_{A}$ et l'application $\partial_{j, A}$ est l'application résidu. 
Lorsque $X$ est propre et lisse, les résultats de Bloch et Ogus permettent d'identifier le groupe $H_{\mathrm{nr}}^{j}\left(X, \mu_{n}^{\otimes i}\right)$ au groupe de cohomologie de Zariski $H^{0}\left(X, \mathscr{H}^{j}\left(\mu_{n}^{\otimes i}\right)\right)$, où $\mathscr{H}^{j}\left(\mu_{n}^{\otimes i}\right)$ désigne le faisceau de Zariski sur $X$ associé au préfaisceau $U \mapsto$ $H^{j}\left(U, \mu_{n}^{\otimes i}\right)$ [Colliot-Thélène 1995].

On note $H^{i}(X, \mathbb{Q} / \mathbb{Z}(j))$ et $H^{i}\left(X, \mathbb{Q}_{l} / \mathbb{Z}_{l}(j)\right)$ la limite inductive des groupes $H^{i}\left(X, \mu_{n}^{\otimes j}\right)$ lorsque $n$ varie respectivement parmi les entiers et parmi les puissances d'un nombre premier $l \neq$ car. $k$.

On définit de même les groupes $H_{\mathrm{nr}}^{i}(X, \mathbb{Q} / \mathbb{Z}(j))$ et $H_{\mathrm{nr}}^{i}\left(X, \mathbb{Q}_{l} / \mathbb{Z}_{l}(j)\right)$, comme limites inductives des groupes $H_{\mathrm{nr}}^{i}\left(X, \mu_{n}^{\otimes j}\right)$.

On note $\mathbb{G}_{m}$ le groupe multiplicatif sur un schéma $X$ et le faisceau étale ainsi défini. On écrit $\operatorname{Br} X=H_{\text {ett }}^{2}\left(X, \mathbb{G}_{m}\right)$ pour le groupe de Brauer cohomologique de $X$ et $\operatorname{Pic}(X)=H_{\text {Zar }}^{1}\left(X, O_{X}^{*}\right) \simeq H_{\text {ét }}^{1}\left(X, \mathbb{G}_{m}\right)$ pour le groupe de Picard.

Rappels de $K$-théorie. Pour $X$ un schéma noethérien et $j$ un entier positif on note $\mathscr{K}_{j}$ le faisceau de Zariski associé au préfaisceau $U \mapsto K_{j}\left(H^{0}\left(U, O_{U}\right)\right)$, le groupe $K_{j}(A)$ étant celui associé par Quillen [1973] à l'anneau $A$.

Lorsque $X$ est une variété lisse sur un corps $k$, la conjecture de Gersten, établie par Quillen, permet de calculer les groupes de cohomologie de Zariski $H^{i}\left(X, \mathscr{K}_{j}\right)$ comme les groupes de cohomologie du complexe de Gersten. Lorsque $j=2$, qui est le cas qui nous intéresse dans la suite, ce complexe s'écrit

$$
K_{2} k(X) \stackrel{d_{2}}{\rightarrow} \bigoplus_{x \in X^{(1)}} k(x)^{*} \stackrel{d_{1}}{\rightarrow} \bigoplus_{x \in X^{(2)}} \mathbb{Z},
$$

où l'application $d_{2}$ est donnée par le symbole modéré et l'application $d_{1}$ est obtenue par la somme des flèches diviseurs après normalisation des variétés considérées. On a ainsi $H^{0}\left(X, \mathscr{K}_{2}\right)=\operatorname{Ker} d_{2}$ et $H^{1}\left(X, \mathscr{K}_{2}\right)=\operatorname{Ker} d_{1} / \operatorname{Im} d_{2}$.

Étant donné un corps $k$, le groupe $K_{2} k$ coïncide avec le groupe de $K$-théorie de Milnor $K_{2}^{M} k$, quotient de $k^{*} \otimes_{\mathbb{Z}} k^{*}$ par le sous-groupe engendré par les éléments $a \otimes b$ avec $a+b=1$.

Cette description permet de voir que pour $X$ une variété lisse sur un corps $k$ on a une flèche naturelle

$$
\operatorname{Pic}(X) \otimes k^{*} \rightarrow H^{1}\left(X, \mathscr{K}_{2}\right) .
$$

En effet, on a le diagramme commutatif suivant :

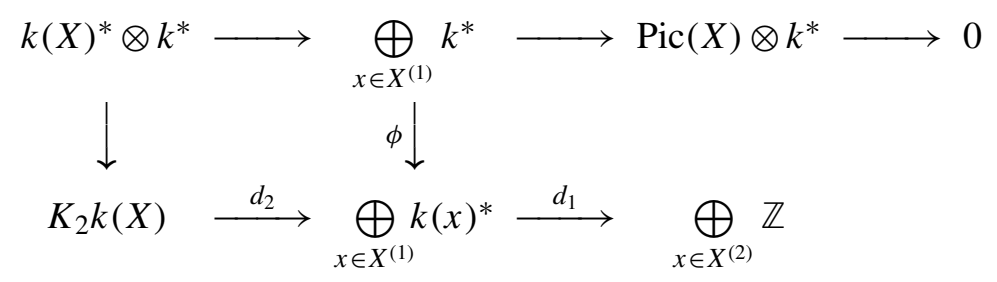


où la première ligne est obtenue à partir de la suite exacte définissant le groupe $\operatorname{Pic}(X)$ par tensorisation avec $k^{*}$. On vérifie que la composé $d_{1} \circ \phi$ vaut zéro, ce qui permet de définir la flèche $\operatorname{Pic}(X) \otimes k^{*} \rightarrow H^{1}\left(X, \mathscr{K}_{2}\right)$ par chasse au diagramme.

\section{Comparaison entre groupes de Chow en codimension deux et cohomologie non ramifiée en degré trois}

Dans cette partie on donne la preuve du théorème suivant :

Théorème 2.1. Soit $X$ une $\mathbb{Q}$-variété projective et lisse, géométriquement rationnelle. Pour presque tout nombre premier $p$, il existe une réduction $X_{p}$ de $X$ modulo $p$ qui est une $\mathbb{F}_{p}$-variété projective et lisse, géométriquement rationnelle, telle que

$$
H_{\mathrm{nr}}^{3}\left(X_{p}, \mathbb{Q}_{l} / \mathbb{Z}_{l}(2)\right) \stackrel{\simeq}{\rightrightarrows} \operatorname{Coker}\left[C H^{2}\left(X_{p}\right) \rightarrow C H^{2}\left(\bar{X}_{p}\right)^{G}\right]\{l\}
$$

pour tout nombre premier $l,(l, p)=1$.

Remarque 2.2. Pour définir $X_{p}$ on choisit un modèle projectif et lisse $\mathscr{Q}$ de $X$ au-dessus d'un ouvert convenable $U \subset \operatorname{Spec} \mathbb{Z},(p) \in U$, et on pose $X_{p}=\mathscr{X} \otimes \mathbb{F}_{p}$. Cette construction dépend du modèle choisi.

Pour démontrer le théorème 2.1, on utilise le résultat suivant :

Théorème 2.3 [Kahn 1996, théorème 1 et corollaire p. 397, partie 1]. Soit $k$ un corps de caractéristique $p \geq 0$, de dimension cohomologique au plus 3. Soit $X$ une $k$-variété projective et lisse. Supposons que

(i) $K_{2} \bar{k} \stackrel{\sim}{\rightarrow} H^{0}\left(\bar{X}, \mathscr{K}_{2}\right)$ et

(ii) le groupe $H_{\mathrm{nr}}^{3}(\bar{X}, \mathbb{Q} / \mathbb{Z}(2))$ est nul, resp. de torsion p-primaire si car. $k>0$. Alors on a une suite exacte naturelle, resp. exacte à la p-torsion près si car. $k>0$

$$
\begin{aligned}
H^{1}\left(k, H^{1}\left(\bar{X}, \mathscr{K}_{2}\right)\right) & \rightarrow \operatorname{Coker}\left[H^{3}(k, \mathbb{Q} / \mathbb{Z}(2)) \rightarrow H_{\mathrm{nr}}^{3}(X, \mathbb{Q} / \mathbb{Z}(2))\right] \rightarrow \\
& \rightarrow \operatorname{Coker}\left[C H^{2}(X) \rightarrow C H^{2}(\bar{X})^{G}\right] \rightarrow H^{2}\left(k, H^{1}\left(\bar{X}, \mathcal{K}_{2}\right)\right) .
\end{aligned}
$$

Remarque 2.4. Voir [Kahn 1996, p. 398] pour la définition des groupes de cohomologie à coefficients dans $\mathbb{Q} / \mathbb{Z}(2)$ en caractéristique positive.

Il est ainsi nécessaire de vérifier les hypothèses (i) et (ii) pour une variété géométriquement rationnelle $X$. Les énoncés suivants, cas particuliers de [Colliot-Thélène 1995], 2.1.9 (cf. aussi 4.1.5), sont bien connus.

Proposition 2.5. Soit $k$ un corps. Soit X une $k$-variété projective et lisse, $k$-rationnelle. Alors

(i) $H_{\mathrm{nr}}^{j}\left(X, \mu_{n}^{\otimes i}\right) \simeq H^{j}\left(k, \mu_{n}^{\otimes i}\right)$ pour tout $j \geq 1$;

(ii) l'application naturelle $K_{2} k \rightarrow H^{0}\left(X, \mathscr{K}_{2}\right)$ est un isomorphisme;

(iii) le groupe $\operatorname{Pic}(X)$ est libre de type fini. 
L'énoncé suivant permet de comprendre le module galoisien $H^{1}\left(\bar{X}, \mathscr{K}_{2}\right)$.

Proposition 2.6. Soit $k$ un corps algébriquement clos de caractéristique nulle. Le noyau $K(X)$ et le conoyau $C(X)$ de l'application $\operatorname{Pic}(X) \otimes k^{*} \rightarrow H^{1}\left(X, \mathscr{K}_{2}\right)$ sont des invariants birationnels des $k$-variétés intègres, projectives et lisses. En particulier, l'application $\operatorname{Pic}(X) \otimes k^{*} \rightarrow H^{1}\left(X, \mathscr{K}_{2}\right)$ est un isomorphisme pour $X$ une variété projective et lisse, $k$-rationnelle.

Démonstration. Le complexe de groupes abéliens

$$
\operatorname{Pic}(X) \otimes k^{*} \rightarrow H^{1}\left(X, K_{2}\right) .
$$

est fonctoriel contravariant pour les morphismes dominants de variétés projectives et lisses. Le noyau $K(X)$ et le conoyau $C(X)$ de $\operatorname{Pic}(X) \otimes k^{*} \rightarrow H^{1}\left(X, K_{2}\right)$ sont alors des foncteurs contravariants pour de tels morphismes. Soit $F$ l'un de ces foncteurs.

Soient $X, Y$ deux variétés intègres, projectives et lisses. Montrons qu'un morphisme birationnel $X \rightarrow Y$ induit un isomorphisme $F(Y) \rightarrow F(X)$. D'après Hironaka, il existe deux $k$-variétés projectives et lisses $X^{\prime}$ et $Y^{\prime}$ et un diagramme commutatif

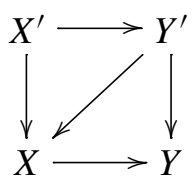

où les flèches verticales sont des suites d'éclatements de centres lisses. D'après le lemme ci-dessous, $F(X)$ est isomorphe à $F\left(X^{\prime}\right)$, respectivement $F(Y)$ est isomorphe à $F\left(Y^{\prime}\right)$. On en déduit par fonctorialité que $F(X)$ est isomorphe à $F(Y)$.

Si maintenant on a une application rationnelle $X \rightarrow Y$, on utilise Hironaka pour trouver une variété projective et lisse $Z$ avec $Z \rightarrow X$ et $Z \rightarrow Y$ deux morphismes birationnels. D'après ce qui précède, $F(X) \simeq F(Z) \simeq F(Y)$. Ainsi $F(X)$ est un invariant birationnel des $k$-variétés intègres, projectives et lisses.

Le fait que $F\left(\mathbb{P}_{k}^{n}\right)=0$ est bien connu. On établit d'abord que $H^{1}\left(\mathbb{A}_{k}^{1}, \mathscr{K}_{2}\right)=0$ et ensuite que $H^{1}\left(\mathbb{A}_{k}^{n}, \mathscr{K}_{2}\right)=0$ par des fibrations successives à fibres $\mathbb{A}^{1}$. L'énoncé pour $\mathbb{P}_{k}^{n}$ s'en suit par récurrence, en se restreignant à l'hyperplan à l'infini.

Remarque 2.7. On peut montrer plus généralement que pour $X$ lisse sur un corps, $H^{i}\left(\mathbb{A}_{X}^{n}, \mathscr{K}_{j}\right)$ est isomorphe à $H^{i}\left(X, \mathscr{K}_{j}\right)$, et donner une expression explicite de $H^{i}\left(\mathbb{P}_{X}^{n}, \mathscr{K}_{j}\right)$ en termes de $K$-cohomologie de $X$. Voir [Sherman 1979].

Lemme 2.8. Soit $k$ un corps algébriquement clos. Soit $X$ une $k$-variété intègre, projective et lisse. Soit $Z \subset X$ une sous-variété intègre, projective et lisse, de codimension au moins 2 et soit $\pi: X^{\prime} \rightarrow X$ l'éclatement de $X$ le long de $Z$. Alors les applications $K(X) \rightarrow K\left(X^{\prime}\right)$ et $C(X) \rightarrow C\left(X^{\prime}\right)$ sont des isomorphismes. 
Démonstration. Soit $Z^{\prime}$ le diviseur exceptionnel de $X^{\prime}$ et soit $U=X \backslash Z \simeq X^{\prime} \backslash Z^{\prime}$. Supposons d'abord que $Z$ est de codimension 2. On a les suites exactes horizontales de complexes verticaux :

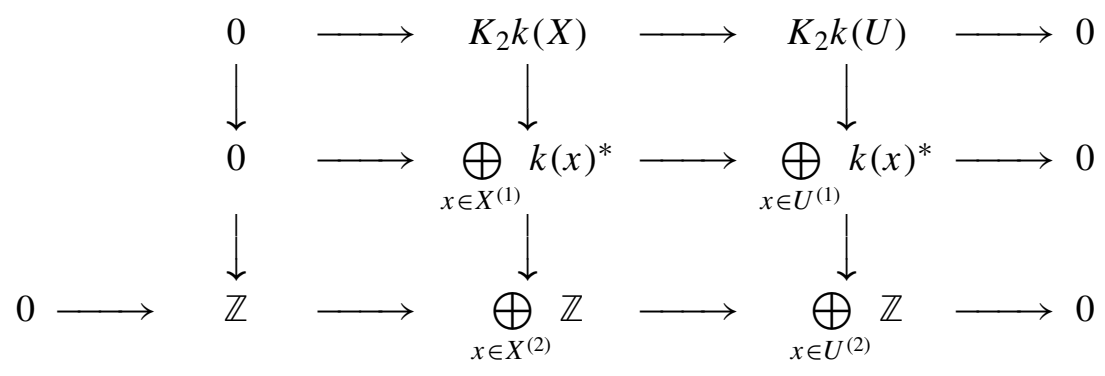

et

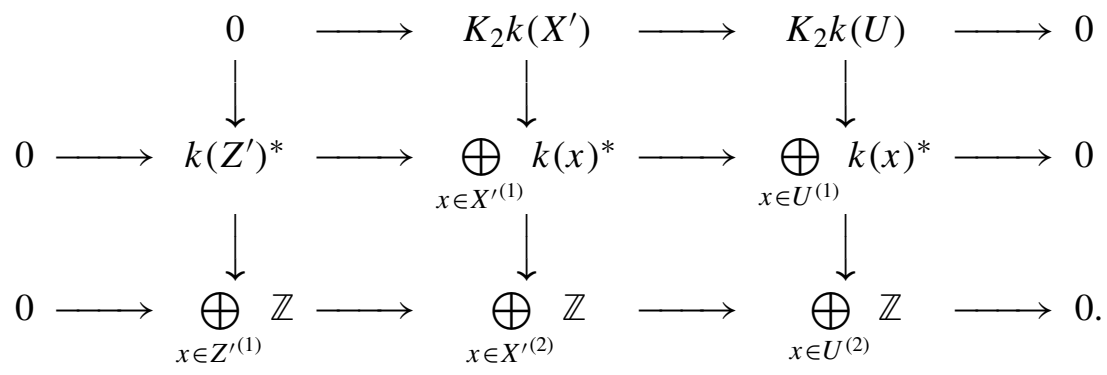

On a ainsi des suites longues induites en cohomologie :

$$
\begin{array}{r}
0 \rightarrow H^{0}\left(X, \mathscr{K}_{2}\right) \rightarrow H^{0}\left(U, \mathscr{K}_{2}\right) \rightarrow 0 \rightarrow H^{1}\left(X, \mathscr{K}_{2}\right) \rightarrow H^{1}\left(U, \mathscr{K}_{2}\right) \rightarrow \mathbb{Z} \rightarrow \\
\rightarrow C H^{2}(X) \rightarrow C H^{2}(U) \rightarrow 0, \quad \text { (3) } \\
0 \rightarrow H^{0}\left(X^{\prime}, \mathscr{K}_{2}\right) \rightarrow H^{0}\left(U, \mathscr{K}_{2}\right) \rightarrow k^{*} \rightarrow H^{1}\left(X^{\prime}, \mathscr{K}_{2}\right) \rightarrow H^{1}\left(U, \mathscr{K}_{2}\right) \rightarrow \operatorname{Pic}\left(Z^{\prime}\right) \rightarrow \\
\rightarrow C H^{2}\left(X^{\prime}\right) \rightarrow C H^{2}(U) \rightarrow 0 .
\end{array}
$$

Dans la suite (3), la flèche $\mathbb{Z} \rightarrow C H^{2}(X)$ est donnée par $1 \mapsto[Z]$. En prenant l'intersection avec un hyperplan général, on voit que cette flèche est injective. Ainsi l'application $H^{1}\left(X, \mathscr{K}_{2}\right) \rightarrow H^{1}\left(U, \mathscr{K}_{2}\right)$ est un isomorphisme.

Si $Z$ est de codimension plus grande que 2, on a encore la suite (4) et les groupes $H^{1}\left(X, \mathscr{K}_{2}\right)$ et $H^{1}\left(U, \mathscr{K}_{2}\right)$ sont isomorphes, car ils ne dependent que de points de codimension au plus 2 .

Par fonctorialité, on a le diagramme commutatif suivant :

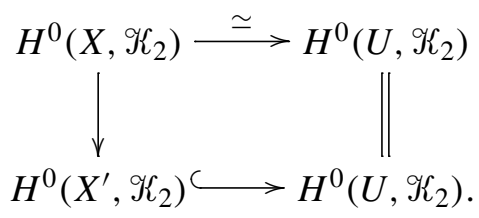


Ainsi l'application $H^{0}\left(X^{\prime}, \mathscr{K}_{2}\right) \rightarrow H^{0}\left(U, \mathscr{K}_{2}\right)$ est un isomorphisme. En utilisant la suite (4), on obtient le diagramme commutatif de suites exactes suivant :

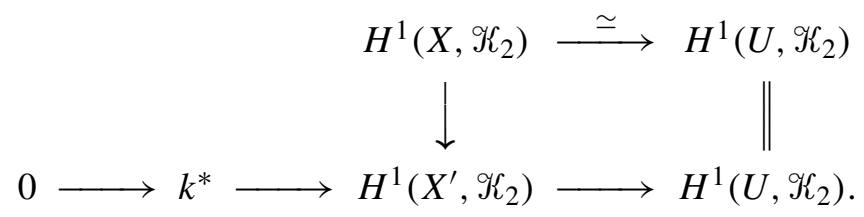

On a donc une suite exacte scindée :

$$
0 \rightarrow k^{*} \rightarrow H^{1}\left(X^{\prime}, \mathscr{K}_{2}\right) \rightarrow H^{1}\left(X, \mathscr{K}_{2}\right) \rightarrow 0 .
$$

Ainsi $H^{1}\left(X^{\prime}, \mathscr{K}_{2}\right) \simeq H^{1}\left(X, \mathscr{K}_{2}\right) \oplus k^{*}$. Puisque $\operatorname{Pic}\left(X^{\prime}\right)=\operatorname{Pic}(X) \oplus \mathbb{Z} \cdot\left[Z^{\prime}\right]$, on en déduit l'énoncé du lemme.

Remarque 2.9. En utilisant l'action des correspondances sur les groupes de Chow supérieurs, on peut établir la proposition 2.6 en toute caractéristique. Cela n'est pas nécessaire pour la démonstration du théorème 2.1 .

Preuve du théorème 2.1. Puisque $X$ est une $\mathbb{Q}$-variété géométriquement rationnelle, il existe une extension finie $K / \mathbb{Q}$ et une $K$-variété projective et lisse $Z$, deux morphismes $K$-birationnels $Z \rightarrow X_{K}$ et $Z \rightarrow \mathbb{P}_{K}^{n}$, et deux diagrammes commutatifs

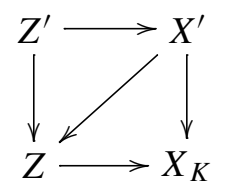

et

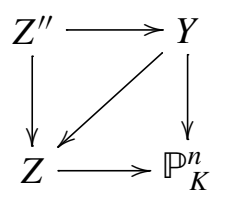

où les flèches verticales sont des suites d'éclatements de centres lisses. Pour presque toute place $v$ de $K$, les centres d'éclatements admettent des réductions lisses et les diagrammes (5) induisent des diagrammes analogues sur le corps résiduel $k(v)$. Ainsi, pour presque toute place $v$ de $K$, on peut définir une réduction $X_{p}$ de $X$ modulo $p, p=$ car. $k(v)$, qui est une $\mathbb{F}_{p}$-variété projective et lisse, géométriquement rationnelle et des réductions de $Z, Z^{\prime}, Z^{\prime \prime}, X$ et $Y$ sur $k(v)$, qui sont des variétés lisses et telles qu'on a des diagrammes commutatifs sur $\overline{\mathbb{F}}_{p}$
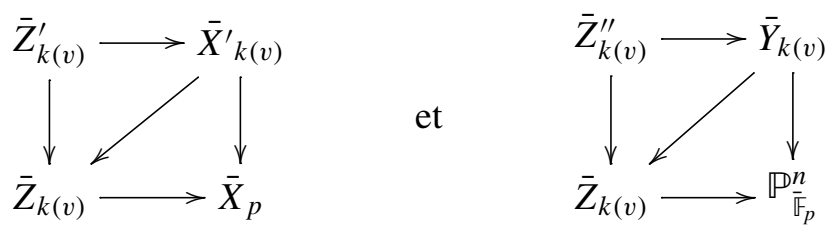

où les flèches verticales sont des suites d'éclatements de centres lisses. En appliquant le lemme 2.8, on déduit que l'application $\operatorname{Pic}\left(\bar{X}_{p}\right) \otimes \overline{\mathbb{F}}_{p}^{*} \rightarrow H^{1}\left(\bar{X}_{p}, \mathscr{K}_{2}\right)$ est un isomorphisme (voir aussi la proposition 2.6). 
Montrons que les hypothèses du théorème 2.3 sont satisfaites pour une telle réduction $X_{p}$. D'après la proposition $2.5, K_{2} \overline{\mathbb{F}}_{p} \stackrel{\sim}{\rightarrow} H^{0}\left(\bar{X}_{p}, \mathscr{K}_{2}\right)=0$ car $X_{p}$ est géométriquement rationnelle. De même, $H_{\mathrm{nr}}^{3}\left(\bar{X}_{p}, \mathbb{Q}_{l} / \mathbb{Z}_{l}(2)\right)=H^{3}\left(\overline{\mathbb{F}}_{p}, \mathbb{Q}_{l} / \mathbb{Z}_{l}(2)\right)=$ 0 car $\overline{\mathbb{F}}_{p}$ est séparablement clos.

Montrons ensuite que le groupe $H^{i}\left(\mathbb{F}_{p}, H^{1}\left(\bar{X}_{p}, \mathscr{K}_{2}\right)\right) \simeq H^{i}\left(\mathbb{F}_{p}, \operatorname{Pic}\left(\bar{X}_{p}\right) \otimes \overline{\mathbb{F}}_{p}^{*}\right)$ est nul pour tout $i \geq 1$. D'après la proposition 2.5, le $\mathbb{Z}$-module $\operatorname{Pic}\left(\bar{X}_{p}\right)$ est libre de type fini. Considérons une extension finie galoisienne $L / \mathbb{F}_{p}$ qui déploie $\operatorname{Pic}\left(\bar{X}_{p}\right)$. Considérons la suite de restriction-inflation :

$0 \rightarrow H^{1}\left(\operatorname{Gal}\left(L / \mathbb{F}_{p}\right), \operatorname{Pic} \bar{X}_{p, L} \otimes L^{*}\right) \rightarrow$

$$
H^{1}\left(\mathbb{F}_{p}, \operatorname{Pic} \bar{X}_{p} \otimes \mathbb{F}_{p}^{*}\right) \rightarrow H^{1}\left(\operatorname{Gal}\left(\overline{\mathbb{F}}_{p} / L\right), \operatorname{Pic} \bar{X}_{p} \otimes \overline{\mathbb{F}}_{p}^{*}\right) .
$$

On a $H^{1}\left(\operatorname{Gal}\left(\overline{\mathbb{F}}_{p} / L\right), \operatorname{Pic} \bar{X}_{p} \otimes \overline{\mathbb{F}}_{p}^{*}\right)=0$ d'après le théorème de Hilbert 90 . Puisque la dimension cohomologique de $\mathbb{F}_{p}$ est $1, H^{1}\left(\operatorname{Gal}\left(L / \mathbb{F}_{p}\right), \operatorname{Pic} X_{p, L} \otimes L^{*}\right)=0$ [Serre 1968, p. 170]. On a donc $H^{i}\left(\mathbb{F}_{p}, \operatorname{Pic}\left(\bar{X}_{p}\right) \otimes \overline{\mathbb{F}}_{p}^{*}\right)=0$ pour tout $i \geq 1$.

Notons que $H^{3}\left(\mathbb{F}_{p}, \mathbb{Q}_{l} / \mathbb{Z}_{l}(2)\right)=0$ car la dimension cohomologique de $\mathbb{F}_{p}$ est 1 . La suite (2) donne alors un isomorphisme :

$$
H_{\mathrm{nr}}^{3}\left(X_{p}, \mathbb{Q}_{l} / \mathbb{Z}_{l}(2)\right) \stackrel{\sim}{\rightarrow} \operatorname{Coker}\left[C H^{2}\left(X_{p}\right) \rightarrow C H^{2}\left(\bar{X}_{p}\right)^{G}\right]\{l\} .
$$

Remarque 2.10. Pour établir $H^{i}\left(k, H^{1}\left(\bar{X}, \mathscr{K}_{2}\right)\right)=0, i=1,2$ pour $X$ une variété projective et lisse, géométriquement rationnelle, définie sur un corps fini $k$, on aurait pu faire appel à des résultats généraux sur les variétés projectives et lisses [Colliot-Thélène et Raskind 1985, 2.12 et 2.14; Gros et Suwa 1988, 4.1]. Ces résultats généraux réposent en particulier sur les conjectures de Weil (démontrées par Deligne). Pour ce dont on a besoin dans la suite, le théorème 2.1 suffit.

\section{L'exemple}

Dans cette partie, pour une infinité de nombres premiers $p$, on construit une variété projective et lisse géométriquement rationnelle $X$, définie sur le corps fini $\mathbb{F}_{p}$, telle que l'application

$$
C H^{2}(X) \rightarrow C H^{2}(\bar{X})^{G}
$$

n'est pas surjective.

Remarque 3.1. Si $X$ est une variété projective et lisse, géométriquement intègre, définie sur le corps fini $\mathbb{F}_{p}$, l'application $\mathrm{CH}^{i}(X) \rightarrow C H^{i}(\bar{X})^{G}$ est surjective pour $i=0,1, d$. Le cas $i=0$ est immédiat. Pour $i=1, \operatorname{Pic}(X) \stackrel{\sim}{\rightarrow} C H^{1}(X)$ car $X$ est lisse. Puisque $X$ est projective et géométriquement intègre, $\bar{k}[X]^{*}=\bar{k}^{*}$ et la suite spectrale $E_{2}^{p q}=H^{p}\left(G, H^{q}\left(\bar{X}, \mathbb{G}_{m}\right)\right) \Rightarrow H^{p+q}\left(X, \mathbb{G}_{m}\right)$ donne une suite exacte

$$
0 \rightarrow \operatorname{Pic}(X) \rightarrow \operatorname{Pic}(\bar{X})^{G} \rightarrow H^{2}\left(G, \bar{k}^{*}\right) \rightarrow \operatorname{Br} X .
$$


Puisque le groupe $H^{2}\left(G, \bar{k}^{*}\right)=\operatorname{Br} k$ est nul pour un corps fini, on a la surjectivité pour $i=1$. Plus généralement, il en est ainsi pour toute variété $X$ projective et lisse, géométriquement intègre, avec un point rationnel, définie sur un corps $k$ quelconque : pour une telle variété l'application $H^{2}\left(G, \bar{k}^{*}\right) \rightarrow \operatorname{Br} X$ est injective.

Pour $i=d$, c'est-à-dire dans le cas de zéro-cycles, on sait que $X$ possède un zéro-cycle de degré 1 d'après les estimations de Lang et Weil [1954]. Il suffit donc de voir que l'application entre les groupes de Chow de zéro-cycles de degré zéro $A_{0}(X) \rightarrow A_{0}(\bar{X})^{G}$ est surjective. Ceci résulte de la comparaison de ces derniers groupes avec les points rationnels (resp. les $\overline{\mathbb{F}}_{p}$-points) de la variété d'Albanese $\operatorname{Alb}_{X}$ de $X$. En effet, l'application $A_{0}(X) \rightarrow \operatorname{Alb}_{X}\left(\mathbb{F}_{p}\right)$ est surjective [Kato et Saito 1983, proposition 9, p. 274], et l'application $A_{0}(\bar{X}) \rightarrow \operatorname{Alb}_{X}\left(\overline{\mathbb{F}}_{p}\right)$ est un isomorphisme [Rojtman 1980; Milne 1982].

D'après le théorème 2.1 , si $X$ est géométriquement rationnelle, il suffit d'assurer que le groupe $H_{\mathrm{nr}}^{3}\left(X, \mathbb{Q}_{l} / \mathbb{Z}_{l}(2)\right)$ est non nul pour un certain nombre premier $l$, $l \neq p$. Colliot-Thélène et Ojanguren [1989] construisent de tels exemples sur le corps des complexes pour $l=2$. Les variétés ainsi construites sont unirationnelles (c'est-à-dire, dominées par un ouvert de l'espace projectif). Via la proposition 2.5, on obtient ainsi des exemples de variétés unirationnelles non rationnelles. Dans la suite, on utilise la même méthode pour produire des exemples sur les corps finis.

La stratégie est la suivante :

(1) On considère une quadrique projective et lisse $Q$ sur le corps $F=\mathbb{F}_{p}(x, y)$, $p \neq 2$, définie dans $\mathbb{P}_{F}^{4}$ par une équation homogène

$$
x_{0}^{2}-a x_{1}^{2}-f x_{2}^{2}+a f x_{3}^{2}-g_{1} g_{2} x_{4}^{2}=0
$$

où $a \in \mathbb{F}_{p}$ est une constante et $f, g_{1}, g_{2} \in F$. La quadrique $Q$ admet un point rationnel sur $\overline{\mathbb{F}}_{p}(x, y)$, elle est donc $\overline{\mathbb{F}}_{p}(x, y)$-rationnelle.

(2) On donne des conditions nécessaires sur les coefficients dans (6) pour que le cup-produit $\left(a, f, g_{1}\right)$ soit non nul dans $H_{\mathrm{nr}}^{3}(Q, \mathbb{Z} / 2)$.

(3) On vérifie que l'on peut trouver $a \in \mathbb{Z}$ et $f, g_{1}, g_{2} \in \mathbb{Z}(x, y)$ tels que leurs réductions modulo $p$ vérifient les conditions de l'étape précédente pour le corps $\mathbb{F}_{p}$ pour une infinité de nombres premiers $p$. Par Hironaka, on trouve une variété projective et lisse $X$ définie sur $\mathbb{Q}$, admettant une fibration sur $\mathbb{P}_{\mathbb{Q}}^{2}$ de fibre générique la quadrique définie par (6). Pour presque tout $p, X$ admet une réduction $X_{p}$ modulo $p$ qui est lisse sur $\mathbb{F}_{p}$ et pour une infinité de premiers $p$ le groupe $H_{\mathrm{nr}}^{3}\left(X_{p}, \mathbb{Z} / 2\right)$ est ainsi non nul.

Cohomologie des quadriques. Soit $k$ un corps, car. $k \neq 2$. Soit $\phi$ une forme quadratique non dégénérée de dimension $m$ définie sur $k$. On note $X_{\phi}$ la quadrique projective et lisse dans $\mathbb{P}_{k}^{m-1}$ définie par $\phi$. On appelle $n$-forme de Pfister sur $k$ 
une forme quadratique de type $\left\langle 1,-a_{1}\right\rangle \otimes \cdots \otimes\left\langle 1,-a_{n}\right\rangle, a_{i} \in k^{*}$. Une forme quadratique non dégénérée $\phi$ est dite «voisine de Pfister» s'il existe une forme de Pfister $\phi^{\prime}$ sur $k$ et $a \in k^{*}$ tels que $\phi$ soit une sous-forme de $a \phi^{\prime}$ et que la dimension de $\phi$ soit strictement supérieure à la moitié de la dimension de $\phi^{\prime}$.

Théorème 3.2 [Arason 1975]. Soit $k$ un corps, car. $k \neq 2$. Soit $\phi$ une forme quadratique définie sur $k$, voisine d'une 3-forme de Pfister $\left\langle 1,-a_{1}\right\rangle \otimes\left\langle 1,-a_{2}\right\rangle \otimes\left\langle 1,-a_{3}\right\rangle$. Alors

$$
\operatorname{ker}\left[H^{3}(k, \mathbb{Z} / 2) \rightarrow H^{3}\left(k\left(X_{\phi}\right), \mathbb{Z} / 2\right)\right]=\mathbb{Z} / 2\left(a_{1}, a_{2}, a_{3}\right),
$$

chaque $a_{i}$ étant identifié à sa classe dans $H^{1}(k, \mathbb{Z} / 2) \simeq k^{*} / k^{* 2}$.

Soit $Q$ la quadrique définie sur le corps $F=\mathbb{F}_{p}(x, y), p \neq 2$, par l'équation homogène (6). D'après le théorème d'Arason,

$$
\operatorname{ker}\left[H^{3}(F, \mathbb{Z} / 2) \rightarrow H^{3}(F(Q), \mathbb{Z} / 2)\right]=\mathbb{Z} / 2\left(a, f, g_{1} g_{2}\right) .
$$

Pour trouver un élément non nul dans $H_{\mathrm{nr}}^{3}(Q, \mathbb{Z} / 2)$, on peut ainsi essayer de chercher un élément de $H^{3}(F, \mathbb{Z} / 2)$, différent de $\left(a, f, g_{1} g_{2}\right)$ et qui devient non ramifié dans $H^{3}(F(Q), \mathbb{Z} / 2)$. On va choisir les éléments $a, f, g_{1}$ et $g_{2}$ pour que l'élément $\left(a, f, g_{1}\right)$ convienne.

Faisons d'abord quelques rappels sur les calculs de résidus.

Proposition 3.3 [Colliot-Thélène et Ojanguren 1989, 1.3 et 1.4]. Soit A un anneau de valuation discrète, de corps des fractions $K$ et de corps résiduel $k$. Soit $j \geq 1$ un entier.

(1) Soit $\alpha \in H^{j}(A, \mathbb{Z} / 2)$ et soit $\alpha_{0} \in H^{j}(k, \mathbb{Z} / 2)$ son image par l'application de réduction. Soit $b \in K^{*}$ de valuation $m$ dans $A$ et soit $\beta$ la classe de $b$ dans $H^{1}(K, \mathbb{Z} / 2)$. Alors $\partial_{A}(\alpha \cup \beta)=m \alpha_{0}$.

(2) Soit $\alpha \in H^{j}(K, \mathbb{Z} / 2)$ et soit $b \in A^{*}$ dont la classe est un carré dans $k$. Soit $\beta$ la classe de $b$ dans $H^{1}(K, \mathbb{Z} / 2)$. Alors $\partial_{A}(\alpha \cup \beta)=0$.

On décrit ensuite les conditions qu'on va imposer sur les coefficients de la quadrique $Q$ :

Proposition 3.4. Soit $F=\mathbb{F}_{p}(x, y)$ le corps des fractions rationnelles à deux variables sur le corps fini $\mathbb{F}_{p}$. Soit $a \in \mathbb{F}_{p}^{*} \backslash \mathbb{F}_{p}^{* 2}$ et soient $f, g_{1}, g_{2} \in F$ non nuls. Soit $Q$ la quadrique lisse dans $\mathbb{P}_{F}^{4}$ d'équation homogène

$$
x_{0}^{2}-a x_{1}^{2}-f x_{2}^{2}+a f x_{3}^{2}-g_{1} g_{2} x_{4}^{2}=0 .
$$

\section{Supposons}

(1) pour tout $i=1,2$, il existe un anneau de valuation discrète $B_{i}$ de corps des fractions $F$, tel que $\partial_{B_{i}}\left(a, f, g_{i}\right) \neq 0$; 
(2) pour tout anneau de valuation discrète $B$ de corps des fractions $F$, associé à un point de codimension 1 de $\mathbb{P}_{\mathbb{F}_{p}}^{2}$, soit $\partial_{B}\left(a, f, g_{1}\right)=0$, soit $\partial_{B}\left(a, f, g_{2}\right)=0$;

(3) pour tout anneau de valuation discrète $B$ de corps des fractions $F$, centré en un point fermé $M$ de $\mathbb{P}_{\mathbb{F}_{p}}^{2}$, quitte à la multiplier par un carré dans $F^{*}$, l'une au moins des fonctions $f, g_{1}, g_{2}$ est inversible en $M$.

Alors l'image $\xi_{F(Q)} d u$ cup-produit $\xi=\left(a, f, g_{1}\right)$ dans $H^{3}(F(Q), \mathbb{Z} / 2)$ est un élément non nul de $H_{\mathrm{nr}}^{3}(Q, \mathbb{Z} / 2)$.

Démonstration. Notons d'abord que $\left(a, f, g_{1}\right)$ est non nul dans $H^{3}(F(Q), \mathbb{Z} / 2)$. Sinon, d'après le théorème 3.2 , on a soit $\left(a, f, g_{1}\right)=0$, soit $\left(a, f, g_{1}\right)=\left(a, f, g_{1} g_{2}\right)$. Ainsi soit $\left(a, f, g_{1}\right)=0$, soit $\left(a, f, g_{2}\right)=0$, contradiction avec la condition 1 .

Montrons que pour tout anneau de valuation discrète $B$ de $F$

$$
\text { soit } \partial_{B}\left(a, f, g_{1}\right)=0 \text {, soit } \partial_{B}\left(a, f, g_{2}\right)=0 \text {. }
$$

Pour un tel anneau $B$ on dispose d'un morphisme Spec $B \rightarrow \mathbb{P}_{\mathbb{F}_{p}}^{2}$ et les cas 2 et 3 correspondent à deux possibilités pour l'image du point fermé de $B$. La condition (2) assure $(*)$ si cette image est un point de codimension $1 \mathrm{de} \mathbb{P}_{\mathbb{F}_{p}}^{2}$. Sinon l'image du point $\operatorname{Spec} k_{B}$ est un point fermé $M$ de $\mathbb{P}_{\mathbb{F}_{p}}^{2}$. Soit $\bigcirc_{M}$ l'anneau local de $M$, son corps des fractions est $F$. On dispose d'un morphisme d'anneaux $\mathrm{O}_{M} \rightarrow B$.

On peut supposer, sans perte de généralité, que la fonction $g_{1}$ est inversible dans $\mathrm{O}_{M}$, quitte à la multiplier par un carré. Ainsi la fonction $g_{1}$ est inversible dans $B$. Soit $m$ la valuation de $f$ dans $B$. D'après la proposition 3.3.1, on a

$$
\partial_{B}\left(a, f, g_{1}\right)=\partial_{B}\left(g_{1}, a, f\right)=m\left(\bar{g}_{1}, \bar{a}\right),
$$

où l'on note $\bar{g}_{1}$ et $\bar{a}$ les classes de $g_{1}$ et de $a$ dans $H^{1}\left(k_{B}, \mathbb{Z} / 2\right)$. Comme $g_{1}$ et $a$ sont inversibles dans $\mathrm{O}_{M}$, ces dernières classes proviennent de classes dans $H^{1}\left(k_{M}, \mathbb{Z} / 2\right)$. Ainsi $\left(\bar{g}_{1}, \bar{a}\right)$ provient d'un élément de $H^{2}\left(k_{M}, \mathbb{Z} / 2\right)$. Ce dernier groupe est nul, car $k_{M}$, étant un corps fini, est de dimension cohomologique 1. Ainsi $\partial_{B}\left(a, f, g_{1}\right)=0$.

Montrons maintenant que $\xi_{F(Q)}$ est non ramifié. Soit $A$ un anneau de valuation discrète de $F(Q)$ de corps résiduel $k_{A}$. Si $A$ contient $F$, alors $\xi_{F(Q)}$ provient d'un élément de $H^{3}(A, \mathbb{Z} / 2)$ et son résidu est donc nul. Supposons que $A$ ne contient pas $F$. Alors $B=A \cap F$ est un anneau de valuation discrète de $F$. Soit $k_{B}$ son corps résiduel. On a le diagramme commutatif suivant [Colliot-Thélène et Ojanguren $1989, \S 1]$ :

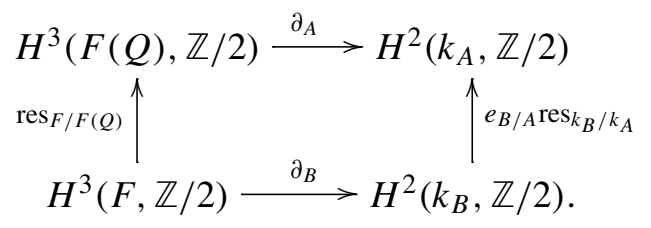


D'après ce qui précède, $\partial_{B}\left(a, f, g_{i}\right)=0$ pour $i=1$ ou pour $i=2$. Si $\partial_{B}\left(a, f, g_{1}\right)=0$, alors $\partial_{A}\left(a, f, g_{1}\right)=0$ d'après le diagramme (8). Supposons que $\partial_{B}\left(a, f, g_{2}\right)=0$. Ainsi $\partial_{A}\left(a, f, g_{2}\right)=0$. Comme $\left(a, f, g_{1} g_{2}\right)$ est nul dans $H^{3}(F(Q), \mathbb{Z} / 2)$, son résidu l'est aussi dans $H^{2}\left(k_{A}, \mathbb{Z} / 2\right)$. On a donc

$$
\partial_{A}\left(a, f, g_{1}\right)=\partial_{A}\left(a, f, g_{1} g_{2}\right)-\partial_{A}\left(a, f, g_{2}\right)=0 .
$$

Ainsi $\xi_{F(Q)}$ est non ramifié.

Construction explicite. On procède maintenant à la construction des exemples.

Soit $k$ un corps. Dans la suite, on va prendre $k=\mathbb{F}_{p}$ ou $k=\mathbb{Q}$. On fixe $x, y, z$ des coordonnées homogènes pour $\mathbb{P}_{k}^{2}$. Soit $a \in k^{*} \backslash k^{* 2}$. Soient $b_{i}, c_{i}, d_{i} \in k^{*} \backslash\{-1\}$, $i=1$, 2, et soit $l_{i}=b_{i} x+c_{i} y+d_{i} z$. Soient $h_{j}, j=1, \ldots, 8$, les formes linéaires $e_{x} x+e_{y} y+e_{z} z, e_{x}, e_{y}, e_{z} \in\{0,1\}$.

On choisit $b_{i}, c_{i}, d_{i}$ de sorte que :

(i) Les droites dans $\mathbb{P}_{k}^{2}$ données par les équations $x=0, y=0, z=0, l_{i}+h_{j}=0$, $i=1,2, j=1, \ldots, 8$, soient deux à deux distinctes.

(ii) Pour tous $1 \leq j, j^{\prime} \leq 8$ les trois droites $x=0, l_{1}+h_{j}=0, l_{2}+h_{j^{\prime}}=0$ dans $\mathbb{P}_{k}^{2}$ sont d'intersection vide.

(iii) Pour tous $1 \leq j, j^{\prime} \leq 8$ les trois droites $y=0, l_{1}+h_{j}=0, l_{2}+h_{j^{\prime}}=0$ dans $\mathbb{P}_{k}^{2}$ sont d'intersection vide.

On prend pour $f, g_{1}, g_{2} \in k\left(\mathbb{P}_{k}^{2}\right)$ les éléments suivants :

$$
f=\frac{x}{y}, \quad g_{1}=\frac{\prod_{j}\left(l_{1}+h_{j}\right)}{y^{8}}, \quad g_{2}=\frac{\prod_{j}\left(l_{2}+h_{j}\right)}{z^{8}} .
$$

Remarque 3.5. Soit $h_{j}=e_{x} x+e_{y} y+e_{z} z$. Les droites $x=0, l_{1}+h_{j}=0$ s'intersectent en un seul point $\left[0:\left(d_{1}+e_{z}\right):-\left(c_{1}+e_{y}\right)\right]$. Ainsi les conditions (ii) et (iii) ci-dessus sont équivalentes aux conditions suivantes :

(ii') Les ensembles

$\left\{\left[\left(d_{1}+e_{z}\right):-\left(c_{1}+e_{y}\right)\right], e_{y}, e_{z} \in\{0,1\}\right\}$ et $\left\{\left[\left(d_{2}+e_{z}\right):-\left(c_{2}+e_{y}\right)\right], e_{y}, e_{z} \in\{0,1\}\right\}$ sont d'intersection vide.

(iii') De même,

$\left\{\left[\left(d_{1}+e_{z}\right):-\left(b_{1}+e_{x}\right)\right], e_{x}, e_{z} \in\{0,1\}\right\} \cap\left\{\left[\left(d_{2}+e_{z}\right):-\left(b_{2}+e_{x}\right)\right], e_{x}, e_{z} \in\{0,1\}\right\}=\varnothing$.

Par exemple, pour

$$
l_{1}=x+y+2 z, l_{2}=3 x+3 y+z
$$

il s'agit de vérifier que les ensembles $\{[2:-1],[2:-2],[3:-1],[3:-2]\}$ et $\{[1:-3],[1:-4],[2:-3],[2:-4]\}$ sont d'intersection vide. Cette condition est satisfaite pour $k=\mathbb{Q}$ ou $k=\mathbb{F}_{p}$ un corps fini avec $p \geq 13$. 
Proposition 3.6. Soit $F=\mathbb{F}_{p}(x, y)$ le corps des fractions rationnelles à deux variables sur le corps fini $\mathbb{F}_{p}$. Soit $Q$ la quadrique lisse dans $\mathbb{P}_{F}^{4}$ d'équation homogène

$$
x_{0}^{2}-a x_{1}^{2}-f x_{2}^{2}+a f x_{3}^{2}-g_{1} g_{2} x_{4}^{2}=0
$$

avec $a \in \mathbb{F}_{p}^{*} \backslash \mathbb{F}_{p}^{* 2}$ et $f, g_{1}, g_{2}$ définis comme dans (9) pour $k=\mathbb{F}_{p}$. Alors le groupe $H_{\mathrm{nr}}^{3}(Q, \mathbb{Z} / 2)$ est non nul.

Démonstration. Notons $A_{x}\left(A_{y}, A_{z}, B_{i, j}\right.$, où $i=1,2$ et $\left.j=1, \ldots, 8\right)$ l'anneau de valuation discrète associé au point générique de la droite $x=0(y=0, z=0$, $\left.l_{i}+h_{j}=0\right)$.

Il s'agit de vérifier les conditions 1, 2 et 3 de la proposition 3.4. Soit $B$ un anneau de valuation discrète de $F$. On a les cas suivants à considérer :

$\underline{B}$ correspond à un point de codimension 1 de $\mathbb{P}_{\mathbb{F}_{p}}^{2}$.

(a) Si $B$ est différent de $A_{x}, A_{y}, A_{z}, B_{i, j}$, le résidu $\partial_{B}\left(a, f, g_{i}\right), i=1,2$, est nul, puisque les fonctions $a, f, g_{1}, g_{2}$ sont inversibles dans un tel anneau $B$.

(b) $B=B_{i, j}$. Si $r \neq i, r=1,2$, alors $\partial_{B_{r, j}}\left(a, f, g_{i}\right)=0$ comme le cas précédent. Fixons $i \in\{1,2\}$. Montrons que $\partial_{B_{i, j}}\left(a, f, g_{i}\right) \neq 0$. Supposons $h_{j}=0$, les autres cas sont identiques. Soit $k$ le corps résiduel de $B_{i, 0}$, i.e. le corps des fonctions de la droite $b_{i} x+c_{i} y+d_{i} z=0, b_{i}, c_{i}, d_{i} \in k^{*}$ (pour $h_{j}$ différent de zéro on utilise ainsi l'hypothèse que $b_{i}, c_{i}, d_{i}$ sont différents de -1$)$. D'après la proposition 3.3.1, $\partial_{B_{i, 0}}\left(a, f, g_{i}\right)=\left(a, \frac{x}{y}\right) \in H^{2}(k, \mathbb{Z} / 2)$. Après passage à des coordonnées affines, on est réduit à établir que le cup-produit $(a, x)$ n'est pas nul dans $H^{2}\left(\mathbb{F}_{p}(x), \mathbb{Z} / 2\right)$. On le voit par exemple en appliquant la proposition 3.3.1 à l'anneau de valuation discrète associé à $x=0: a \in \mathbb{F}_{p}$ est non carré.

(c) $B=A_{x}$. Montrons que $\partial_{A_{x}}\left(a, f, g_{i}\right)=0, i=1,2$. Soit $k_{x}$ le corps résiduel de $A_{x}$, i.e. le corps des fonctions de la droite $x=0$. D'après la proposition 3.3.1, $\left.\partial_{A_{x}}\left(a, f, g_{i}\right)=-\partial_{A_{x}}\left(a, g_{i}, f\right)=-\left(a, g_{i, x}\right) \in H^{2}\left(k_{x}, \mathbb{Z} / 2\right)\right)$, où $g_{i, x}$ désigne la fonction induite par $g_{i}$ sur la droite $x=0$. Mais $g_{i, x}$ est un carré dans $k_{x}$, d'où $\partial_{A_{x}}\left(a, f, g_{i}\right)=0$ d'après la proposition 3.3.2.

(d) $B=A_{y}$. Comme dans le cas précédent, $\partial_{A_{y}}\left(a, f, g_{2}\right)=\left(a, g_{2, y}\right)=0$.

(e) $B=A_{z}$. Alors $\partial_{A_{z}}\left(a, f, g_{1}\right)=0$, car les fonctions $a, f, g_{1}$ sont inversibles dans $A_{z}$.

B correspond à un point fermé $M$ de $\mathbb{P}_{\mathbb{F}_{p}}^{2}$.

(a) Si $M$ n'est pas situé sur une des deux droites $x=0, y=0$, alors $f$ est inversible dans $B$.

(b) Si $M$ est situé sur une des deux droites $x=0, y=0$, alors l'une au moins des fonctions $g_{1} \frac{y^{8}}{z^{8}}, g_{2}$ est inversible dans $B$ d'après les hypothèses (ii)-(iii), car le système $x y=0, \prod_{j}\left(l_{1}+h_{j}\right)=0, \prod_{j}\left(l_{2}+h_{j}\right)=0$ n'a pas de solutions. 
On finit par décrire explicitement les exemples énoncés.

Théorème 3.7. Soit $Q$ la quadrique lisse dans $\mathbb{P}_{\mathbb{Q}(x, y)}^{4}$ d'équation homogène

$$
x_{0}^{2}-a x_{1}^{2}-f x_{2}^{2}+a f x_{3}^{2}-g_{1} g_{2} x_{4}^{2}=0
$$

avec $a \in \mathbb{Q}^{*} \backslash \mathbb{Q}^{* 2}$ et $f, g_{1}, g_{2}$ définis comme dans $(9)$ pour $k=\mathbb{Q}$. Soit X un modèle projectif et lisse de $Q$ sur $\mathbb{P}_{\mathbb{Q}}^{2}: X$ est une $k$-variété projective et lisse et admet une fibration sur $\mathbb{P}_{\mathbb{Q}}^{2}$ à fibre générique $Q$. Pour une infinité de nombres premiers $p$, la réduction $X_{p}$ de $X$ modulo $p$ est bien définie et est une $\mathbb{F}_{p}$-variété projective et lisse, telle que:

(i) $H_{\mathrm{nr}}^{3}\left(X_{p}, \mathbb{Z} / 2\right) \neq 0$;

(ii) l'application $\mathrm{CH}^{2}(X) \rightarrow \mathrm{CH}^{2}(\bar{X})^{G}$ n'est pas surjective.

Démonstration. D'après Hironaka, un modèle projectif et lisse $X$ de $Q$ comme dans l'énoncé existe. De plus, pour une infinité de nombres premiers $p$, l'image de $a$ dans $\mathbb{F}_{p}$ n'est pas un carré (par Chebotarev, ou par application de la loi de réciprocité quadratique) et la variété $X$ a bonne réduction en $p: X_{p}$ est lisse. D'après la proposition 3.6, le groupe $H_{\mathrm{nr}}^{3}\left(X_{p}, \mathbb{Z} / 2\right)$ est non nul. Le théorème 2.1 permet de conclure.

\section{Remerciements}

Je tiens à exprimer ma profonde gratitude à mon directeur de thèse à l'Université Paris-Sud (Orsay), Jean-Louis Colliot-Thélène, pour m' avoir suggéré d'utiliser les méthodes de [Colliot-Thélène et Ojanguren 1989] et m'avoir introduite dans le sujet; sans ses nombreux conseils et réponses cet article n'aurait pas pu voir le jour.

\section{References}

[Arason 1975] J. K. Arason, "Cohomologische invarianten quadratischer Formen”, J. Algebra 36:3 (1975), 448-491. MR 52 \#10592 Zbl 0314.12104

[Colliot-Thélène 1995] J.-L. Colliot-Thélène, "Birational invariants, purity and the Gersten conjecture", pp. 1-64 dans $K$-theory and algebraic geometry: connections with quadratic forms and division algebras (Santa Barbara, CA, 1992), édité par B. Jacob et A. Rosenberg, Proc. Sympos. Pure Math. 58, Amer. Math. Soc., Providence, RI, 1995. MR 96c:14016 Zbl 0834.14009

[Colliot-Thélène et Ojanguren 1989] J.-L. Colliot-Thélène et M. Ojanguren, "Variétés unirationnelles non rationnelles: au-delà de l'exemple d'Artin et Mumford”, Invent. Math. 97:1 (1989), 141-158. MR 90m:14012

[Colliot-Thélène et Raskind 1985] J.-L. Colliot-Thélène et W. Raskind, " $\mathscr{Y}_{2}$-cohomology and the second Chow group”, Math. Ann. 270:2 (1985), 165-199. MR 86m:14005 Zbl 0536.14004 
[Gros et Suwa 1988] M. Gros et N. Suwa, "Application d'Abel-Jacobi p-adique et cycles algébriques”, Duke Math. J. 57:2 (1988), 579-613. MR 89h:14006a Zbl 0697.14005

[Kahn 1996] B. Kahn, "Applications of weight-two motivic cohomology", Doc. Math. 1:17 (1996), 395-416. MR 98b:14007 Zbl 0883.19002

[Kato et Saito 1983] K. Kato et S. Saito, "Unramified class field theory of arithmetical surfaces", Ann. of Math. (2) 118:2 (1983), 241-275. MR 86c:14006 Zbl 0562.14011

[Lang et Weil 1954] S. Lang et A. Weil, "Number of points of varieties in finite fields", Amer. J. Math. 76 (1954), 819-827. MR 16,398d Zbl 0058.27202

[Milne 1982] J. S. Milne, “Zero cycles on algebraic varieties in nonzero characteristic: Rojtman's theorem”, Compositio Math. 47:3 (1982), 271-287. MR 85b:14011 Zbl 0506.14006

[Quillen 1973] D. Quillen, "Higher algebraic $K$-theory, I", pp. 85-147 dans Algebraic K-theory, I: Higher K-theories (Seattle, 1972), édité par H. Bass, Lecture Notes in Math. 341, Springer, Berlin, 1973. MR 49 \#2895 Zbl 0292.18004

[Rojtman 1980] A. A. Rojtman, "The torsion of the group of 0-cycles modulo rational equivalence", Ann. of Math. (2) 111:3 (1980), 553-569. MR 81g:14003 Zbl 0504.14006

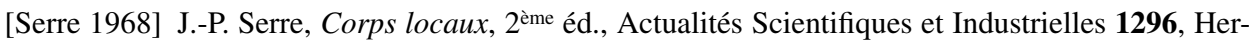
mann, Paris, 1968. MR 50 \#7096 Zbl 0137.02601

[Sherman 1979] C. C. Sherman, "K-cohomology of regular schemes", Comm. Algebra 7:10 (1979), 999-1027. MR 80h:14004 Zbl 0425.18010

Communicated by János Kollár

Received 2010-05-14 Revised 2010-10-12 Accepted 2010-11-15

alena.pirutka@ens.fr École Normale Supérieure, 45 rue d'Ulm, 75230 Paris, France 


\section{Algebra \& Number Theory}

msp.berkeley.edu/ant

\section{EDITORS}

MANAGING EDITOR

Bjorn Poonen

Massachusetts Institute of Technology

Cambridge, USA

\author{
EDITORIAL BOARD CHAIR \\ David Eisenbud \\ University of California \\ Berkeley, USA
}

\section{BOARD OF EDITORS}

Georgia Benkart

Dave Benson

Richard E. Borcherds

John H. Coates

J-L. Colliot-Thélène

Brian D. Conrad

Hélène Esnault

Hubert Flenner

Edward Frenkel

Andrew Granville

Joseph Gubeladze

Ehud Hrushovski

Craig Huneke

Mikhail Kapranov

Yujiro Kawamata

János Kollár

Yuri Manin

Barry Mazur

Philippe Michel

Susan Montgomery
University of Wisconsin, Madison, USA

University of Aberdeen, Scotland

University of California, Berkeley, USA

University of Cambridge, UK

CNRS, Université Paris-Sud, France

University of Michigan, USA

Universität Duisburg-Essen, Germany

Ruhr-Universität, Germany

University of California, Berkeley, USA

Université de Montréal, Canada

San Francisco State University, USA

Hebrew University, Israel

University of Kansas, USA

Yale University, USA

University of Tokyo, Japan

Princeton University, USA

Northwestern University, USA

Harvard University, USA

École Polytechnique Fédérale de Lausanne

University of Southern California, USA
Shigefumi Mori

Raman Parimala

Jonathan Pila

Victor Reiner

Karl Rubin

Peter Sarnak

Joseph H. Silverman

Michael Singer

Ronald Solomon

Vasudevan Srinivas

J. Toby Stafford

Bernd Sturmfels

Richard Taylor

Ravi Vakil

Michel van den Bergh

Marie-France Vignéras

Kei-Ichi Watanabe

Andrei Zelevinsky

Efim Zelmanov
RIMS, Kyoto University, Japan

Emory University, USA

University of Oxford, UK

University of Minnesota, USA

University of California, Irvine, USA

Princeton University, USA

Brown University, USA

North Carolina State University, USA

Ohio State University, USA

Tata Inst. of Fund. Research, India

University of Michigan, USA

University of California, Berkeley, USA

Harvard University, USA

Stanford University, USA

Hasselt University, Belgium

Université Paris VII, France

Nihon University, Japan

Northeastern University, USA

University of California, San Diego, USA

\section{PRODUCTION}

contact@msp.org

Silvio Levy, Scientific Editor

See inside back cover or www.jant.org for submission instructions.

The subscription price for 2011 is US \$150/year for the electronic version, and \$210/year (+\$35 shipping outside the US) for print and electronic. Subscriptions, requests for back issues from the last three years and changes of subscribers address should be sent to Mathematical Sciences Publishers, Department of Mathematics, University of California, Berkeley, CA 94720-3840, USA.

Algebra \& Number Theory (ISSN 1937-0652) at Mathematical Sciences Publishers, Department of Mathematics, University of California, Berkeley, CA 94720-3840 is published continuously online. Periodical rate postage paid at Berkeley, CA 94704, and additional mailing offices.

ANT peer review and production are managed by EditFLOW ${ }^{\circledR}$ from Mathematical Sciences Publishers.

PUBLISHED BY

mathematical sciences publishers

http://msp.org/

A NON-PROFIT CORPORATION

Typeset in IAT $_{\mathrm{E}} \mathrm{X}$

Copyright @2011 by Mathematical Sciences Publishers 


\section{Algebra \& Number Theory}

Volume $5 \quad$ No. $6 \quad 2011$

Higher direct images of the structure sheaf in positive characteristic

ANDRE CHATZISTAMATIOU and KAY RÜLLING

Geometry of quiver Grassmannians of Kronecker type and applications to cluster algebras

Giovanni Cerulli Irelli and Francesco Esposito

Sur le groupe de Chow de codimension deux des variétés sur les corps finis

ALENA PIRUTKA

Sur les invariants d'Iwasawa dans les extensions de Lie $p$-adiques

Guillaume Perbet 